\title{
Arrayed-waveguide grating with wavefront compensation lenses for spatial filter integration
}

\author{
Mitsuhiro Yasumoto ${ }^{\text {a) }}$, Takanori Suzuki, Atsushi Tate, \\ and Hiroyuki Tsuda \\ Department of Electronics and Electrical Engineering, Keio University \\ 3-14-1 Hiyoshi, Kouhoku-ku, Yokohama, Kanagawa, 223-8522, Japan \\ a)yasumoto@tsud.elec.keio.ac.jp
}

\begin{abstract}
We have proposed and fabricated an arrayed-waveguide grating (AWG) with an integrated lens system that is designed to obtain a flat output wavefront. A spatial filter or spatial light modulator could be integrated easily at the output facet of the waveguide. A patterned mirror was attached to the AWG, and doubling of the pulse repetition rate was successfully demonstrated.
\end{abstract}

Keywords: arrayed-waveguide grating, optical planar waveguides

Classification: Photonics devices, circuits, and systems

\section{References}

[1] H. Tsuda, H. Takenouchi, T. Ishii, and C. Amano, "Spectral encoding and decoding of $10 \mathrm{Gbit} / \mathrm{s}$ femtosecond pulses using high resolution arrayed-waveguide grating," Electron. Lett., vol. 35, no. 14, pp. 11861188, July 1999.

[2] A. M. Weiner, "Femtosecond Optical Pulse Shaping And Processing," Prog. Quant. Electr., vol. 19, pp. 161-237, July 1995.

[3] V. J. Hernandez, Y. Du, W. Cong, R. P. Scott, K. Li, J. P. Heritage, Z. Ding, B. H. Kolner, and S. J. B. Yoo, "Spectral Phase Encoded Time Spreading (SPECTS) Code Division Multiple Access for Terabit Optical Access Networks," IEEE J. Lightwave. Technol., vol. 22, no. 11, pp. 2671-2679, Nov. 2004.

[4] T. Kurokawa, H. Tsuda, K. Okamoto, K. Naganuma, H. Takenouchi, Y. Inoue, and M. Ishii, "Time-space-conversion optical signal processing using arrayed-waveguide grating," Electron. Lett., vol. 33, no. 22, pp. 1890-1891, Oct. 1997.

[5] K. Okamoto, Fundamentals of Optical Waveguides, Academic Press, 2000.

[6] M. Yasumoto, T. Suzuki, A. Tate, K. Masuda, and H. Tsuda, "Wavefront Compensation Lens System in an Arrayed-Waveguide Grating for Hybrid Integration of a Phase Encoding Mirror," Frontiers in Optics 2005, FWE4, Oct. 2005.

[7] H. Takenouchi, T. Goh, and T. Ishii, " $2 \times 40$-channel dispersion-slope compensator for 40-Gbit/s WDM transmission systems covering entire C-and L-bands," Opt. Fiber Commun. Conf., vol. 2001, no. March 20, pp. TUS2.1-TUS2.3. 
[8] T. Suzuki and H. Tsuda, " $16 \mathrm{ch}, 100 \mathrm{GHz}$-spacing compact arrayedwaveguide grating using a double bending structure," Proc. Pacific Rim Conf. Lasers and Electro-Optics 2005, Tokyo, Ctuk3-3.

[9] K. Maru, K. Matsui, H. Ishikawa, Y. Abe, S. Kashimura, S. Himi, and H. Uetsuka, "Super-high- $\Delta$ athermal arrayed waveguide grating with resinfilled trenches in slab region," Electron. Lett., vol. 40, no. 6, pp. 374-375, March 2004.

[10] S. Kamei, K. Iemura, A. Kaneko, Y. Inoue, T. Shibata, H. Takahashi, and A. Sugita, "1.5\%- $\Delta$ athermal arrayed-waveguide grating multi/demultiplexer with very low loss groove design," IEEE Photon. Technol. Lett., vol. 17, no. 3, pp. 588-590, March 2005.

[11] K. Mandai, D. Miyamoto, T. Suzuki, H. Tsuda, K. Aizawa, and T. Kurokawa, "Repetition rate and center wavelength-tunable optical pulse generation using an optical comb generator and a high resolution arrayedwaveguide grating," IEEE Photon. Technol. Lett., vol. 18, no. 5, pp. 679681, March 2006.

\section{Introduction}

Various kinds of photonic signal processing, such as dispersion compensation, short pulse generation, pulse shaping, and optical packet generation can be realized by time-to-space conversion and spatial filtering $[1,2,3,4]$. An arrayed-waveguide grating (AWG) [5] is especially suitable for this technique because of its high resolution, simultaneous demultiplexing of all channels, and ease of integration. Since the relative phase between spectral components is crucial, a flat wavefront at the output facet of an AWG is preferable when an external spatial filter is integrated [6]. Therefore, a 90-degree bend mirror has previously been integrated with a slab waveguide in order to provide a flat wavefront [7]; however, setting the mirror tilt was very difficult in this design.

In this paper, we have proposed an integrated lens system that can be incorporated in the slab of the AWG by filling lens-shaped trenches with UVcurable epoxy. This lens system can be used to convert a circular wavefront into a flat one.

\section{Device structure and design}

Fig. 1 (a) shows a schematic of the AWG that was used for spatial filter hybrid integration. To reduce the AWG size, we used V-bend structures in the arrayed-waveguide [8]. The incident light is decomposed into each of its spectral components in the second slab waveguide, and its wavefront is rectified to a flat form by lenses positioned in the output slab waveguide. After the chip is cut-off along the dotted line, a spectrally-decomposed output with a flattened wavefront will be obtained.

The wavefront from each waveguide of the arrayed-waveguide is almost circular and is transformed to a flat wavefront by the lens system; therefore, the focal length of the lens system is set to be equal to the length from the 


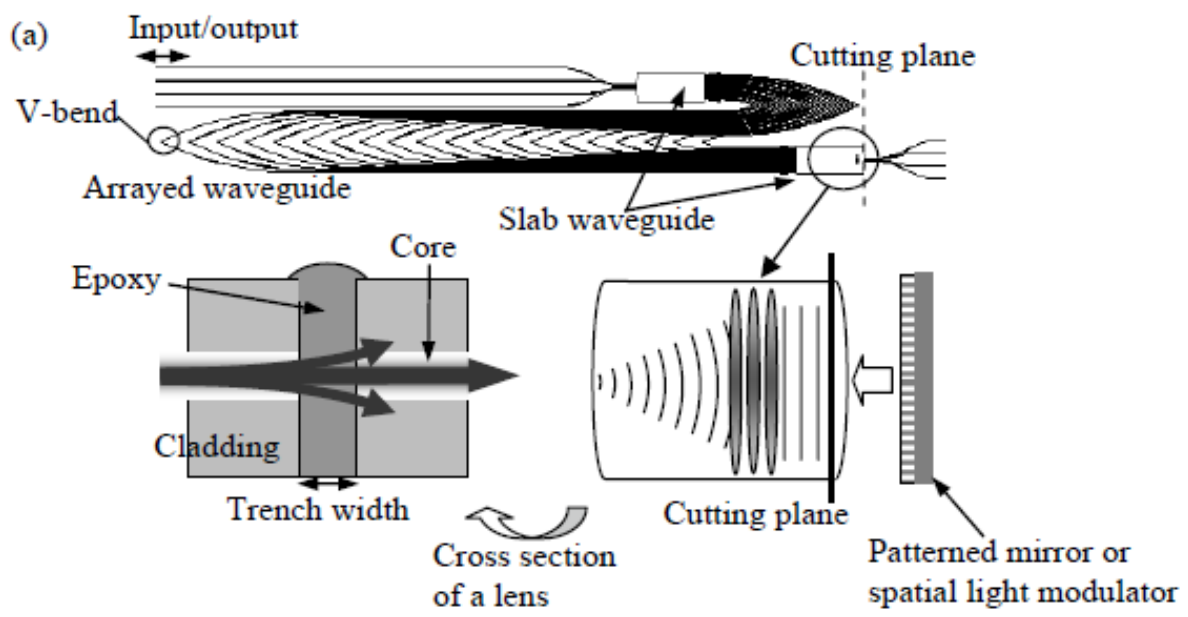

(b)

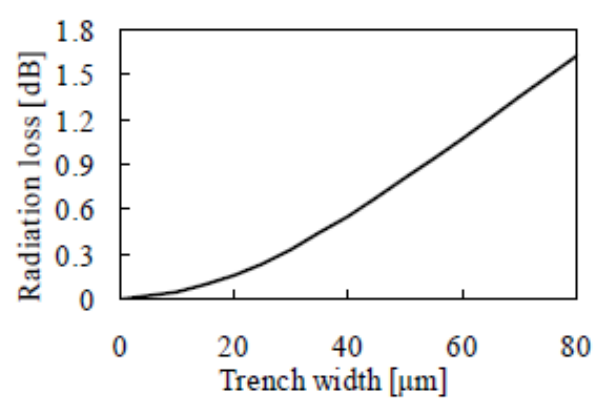

(c)

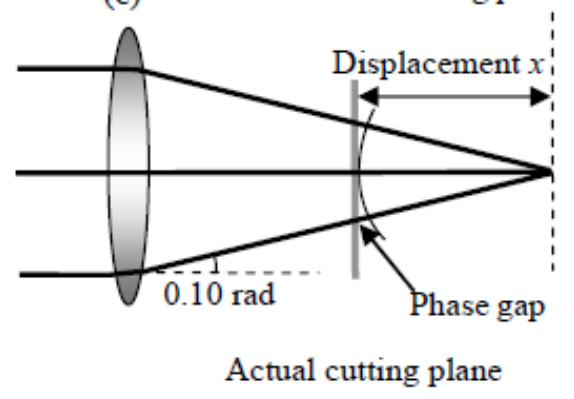

Fig. 1. (a) Schematic of an AWG with wavefront compensation lenses for hybrid integration. The wavefront of the decomposed light is rectified to flat by a convex lens system introduced into the second slab region. Spatial modulators can easily be integrated after cutting the chip along the dotted line. (b) Radiation loss as a function of trench width. (c) Phase error due to the displacement of the cutting plane.

center of the arrayed-waveguide edge to the principal point.

The width of the trench for the lens should be greater than $10 \mu \mathrm{m}$, as determined by the aspect ratio limitation of the etching process. The lens system is fabricated by forming deep trenches using reactive ion etching (RIE) and filling them with UV-curable epoxy. The use of a thick lens leads to large radiation losses, because no vertical confinement structure exists in the lens. However, lower radiation loss can be realized by using a multiple lens system consisting of thinner lenses, rather than using only one thick lens [9, 10]. We have simulated the radiation loss as a function of trench-width by using a beam propagation method (BPM), as shown in Fig. 1(b). We assumed that the trench was filled with the epoxy with the refractive index of 1.51. It can be seen from Fig. 1 (b) that the radiation loss is not in proportion to the width of the trench when the trench width is less than $30 \mu \mathrm{m}$. In this research, we designed three concatenated lenses with a center width of 
$27.7 \mu \mathrm{m}$, a focal length of $8000 \mu \mathrm{m}$, a lens curvature of $636.3 \mu \mathrm{m}$, and a lens interval of $53 \mu \mathrm{m}$. Three lenses are adopted to obtain the focal length of the lens system $(2627 \mu \mathrm{m})$ with minimum number of lenses and the trench width of less than $30 \mu \mathrm{m}$. The distance between the output plane and the center of the lens system was $304 \mu \mathrm{m}$. The diameter of the lens was $214 \mu \mathrm{m}$, such that it was sufficiently greater than the free spatial range of the designed AWG. The refractive indices of the epoxy and slab waveguide were 1.51 and 1.452 , respectively. The BPM simulation showed a relatively low radiation loss of $0.435 \mathrm{~dB}$. The interval of the lenses is optimized to reduce the radiation loss.

The accuracy of the focal length of the lens system is determined by the trench-width errors introduced in the RIE process and by the errors in the refractive index of the lens. We have estimated the distortion of the wavefront by using a ray-tracing method. The wavefront distortion due to the trenchwidth errors was within $0.02 \mathrm{rad}$ for a free spatial range of $144 \mu \mathrm{m}$ when they were $\pm 2 \mu \mathrm{m}$. The wavefront distortion due to the refractive index errors of \pm 0.0005 was within $0.17 \mathrm{rad}$. Therefore, the total distortion of the wavefront was $0.19 \mathrm{rad}$.

Another possible distortion of the wavefront is brought about by the displacement of the cutting plane, as shown in Fig. 1 (c). We have assumed that the maximum angle of the focusing ray is $0.10 \mathrm{rad}$. The calculated distortion of the wavefront is described by

$$
\left[\frac{1}{\cos (0.1)}-1\right] \cdot \frac{2 \pi}{\lambda_{0}} \cdot n_{s} \cdot x \quad[\mathrm{rad}],
$$

where $x$ is the displacement from the target cutting position, $\lambda_{0}$ is a freespace wavelength, and $n_{s}$ is the effective index of slab waveguide. By setting the maximum displacement of the cutting plane to $5 \mu \mathrm{m}$, the phase error was calculated to be $0.15 \mathrm{rad}$.

The overall accuracy of the phase compensation is therefore estimated to be $\pm 0.34 \mathrm{rad}$. This is an acceptable phase error because the phase difference of $0.34 \mathrm{rad}$ is equivalent to $\lambda / 18$, where $\lambda$ is $1.55 \mu \mathrm{m}$.

Table I. Parameters of the AWG and the lens

\begin{tabular}{ll}
\hline \hline Parameter & Value [unit] \\
\hline Core width, core height & $6[\mu \mathrm{m}]$ \\
Relative refractive index difference, $\Delta \mathrm{n}$ & $0.75[\%]$ \\
Center wavelength & $1550.5[\mathrm{~nm}]$ \\
Channel spacing & $12.5[\mathrm{GHz}]$ \\
Number of channels & 8 \\
Diffraction order & 1903 \\
Path difference, $\Delta \mathrm{L}$ & $2035.2[\mu \mathrm{m}]$ \\
Number of arrayed waveguides & 20 \\
Refractive index of lens & 1.51 \\
Focal length (single lens) & $8000[\mu \mathrm{m}]$ \\
Lens curvature & $636.3[\mu \mathrm{m}]$ \\
Width of a lens (center) & $27.7[\mu \mathrm{m}]$ \\
Width of a lens (edge) & $10.2[\mu \mathrm{m}]$ \\
\hline \hline
\end{tabular}


(a)

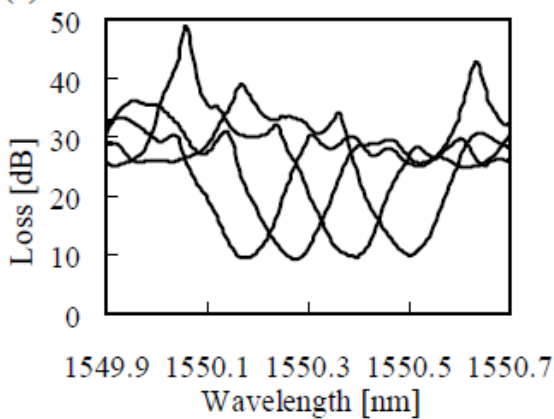

(c)

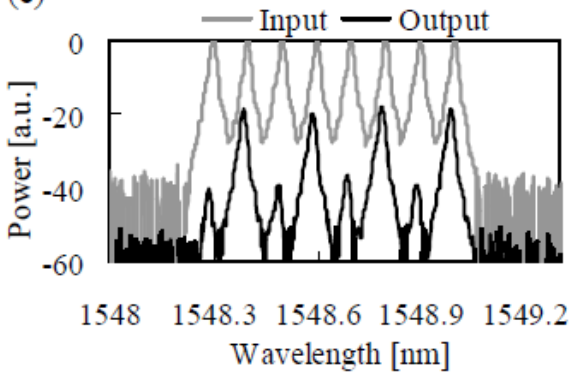

(b)

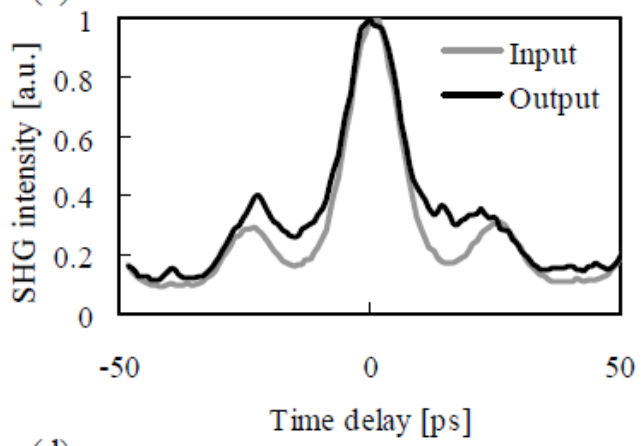

(d)

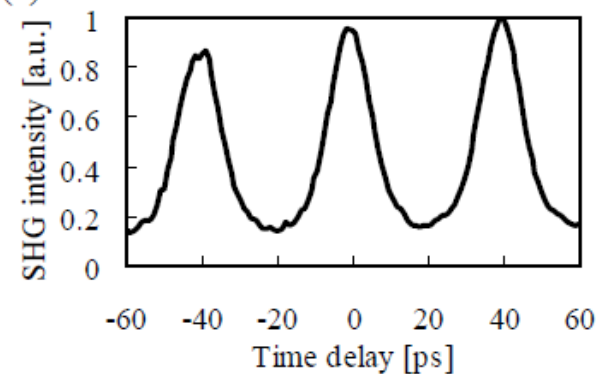

Fig. 2. (a) Spectral characteristics of the fabricated 12.5 GHz-spacing AWG. The four lines correspond to the losses measured with four different temporal output waveguides. (b) Autocorrelation waveform of the input and the reflected pulse trains. (c) $12.5 \mathrm{GHz}$-spacing input optical frequency comb with eight modes, and the output reflected signal. Alternately, four modes were suppressed using a patterned mirror. (d) Autocorrelation waveform of the output reflected signal. The repetition rate of the pulse train was doubled to $25 \mathrm{GHz}$.

\section{Device characteristics and repetition rate doubling demon- stration}

We have designed and fabricated an 8-ch, 12.5-GHz-spacing AWG for hybrid integration. The parameters are shown in Table I. The AWG contains a double-bending structure in the arrayed waveguide region to reduce the chip size [8]. The spectral characteristics were measured after fabricating a lens system in the second slab waveguide, by using 4 temporal output waveguides that were cut-off after measurement. A tunable laser was used as a light source, and the wavelength scan was performed with the resolution of $0.005 \mathrm{~nm}$. The spectral characteristics obtained at the temporal output waveguides are shown in Fig. 2 (a). The insertion loss at the center channel and the polarization dependent loss (PDL) was $9.3 \mathrm{~dB}$ (the higher loss is due to the double-bending structure) and $0.3 \mathrm{~dB}$, respectively. The adjacent crosstalk was $17.4 \mathrm{~dB}$. The chip was diced at several hundred microns from the target line, and gradual polishing was performed. The line dispersion at the output facet was about $0.696 \mathrm{GHz} / \mu \mathrm{m}$. 
Wavefront distortion was measured by attaching a flat mirror to the fabricated AWG and measuring the input and the output optical pulse waveforms. The air gap between the AWG and the mirror was filled with an index matching gel to reduce the Fresnel reflection. The mirror was made of Au deposited onto a silica substrate, and had the same width as the free spatial range of the fabricated AWG. A pulse train consisting of a $12.5 \mathrm{GHz}$-spacing optical frequency comb with eight modes was used as an input. The pulse train was generated by synthesizing a broadband optical frequency comb with an AWG-based optical pulse synthesizer [11]. The input and the output pulses as measured by the autocorrelator are shown in Fig. 2 (b). The relative ratio of the full-width at half maximum (FWHM) between the two pulses was $10.2 \%$, leading to a phase error of $0.35 \mathrm{rad}$ if the circular wavefront distortion was assumed.

Next, we replaced the flat mirror with a stripe mirror such that four channels out of the original eight would be suppressed, as shown in Fig. 2 (c). The stripe mirror had four rectangular segments. Both the width and the interval of the segments were $18.0 \mu \mathrm{m}$ so that the positions of the reflective and non-reflective zones would correspond to the $12.5 \mathrm{GHz}$-spacing of the optical frequency comb. The suppression ratio between the suppressed channels and the non-suppressed channels was about $19 \mathrm{~dB}$. As a result, a pulse train that had a doubled repetition frequency of $25 \mathrm{GHz}$ was successfully generated, as shown in Fig. 2 (d).

\section{Conclusion}

We have proposed an AWG with a flat output wavefront by integrating a convex lens system into a slab waveguide. The AWG was 8-channel, $12.5-\mathrm{GHz}$ spacing and had three lenses in the output slab waveguide. The loss of the lens system was minimized to $0.435 \mathrm{~dB}$. An almost-flat wavefront was confirmed experimentally and the estimated phase error was less than $0.35 \mathrm{rad}$. Repetition-rate doubling of the pulse train was successfully demonstrated by attaching a patterned mirror to the AWG.

\section{Acknowledgments}

This study was supported by a grant from the Japan Science and Technology Corporation, and supported by the Ministry of Public Management, Home Affairs, Posts and Telecommunications. We are grateful to Hitachi Cable, Ltd. for supporting the fabrication of the devices. 